\title{
Complete genome sequence of the kiwifruit bacterial canker pathogen Pseudomonas savastanoi strain MHT1
}

Mingzhao Zhong ${ }^{1,2+}$, Yunhao Sun ${ }^{1,3+}$, Xianzhi Zhang ${ }^{3}$, Hong Liang ${ }^{3,4}$, Lina Xiong ${ }^{5}$ and Qunxin Han ${ }^{1,3,6^{*}}$

\begin{abstract}
Background: Pseudomonas savastanoi is an important plant pathogen that infects and causes symptoms in a variety of economically important crops, causing considerable loss of yield and quality. Because there has been no research reported to date on bacterial canker of kiwifruit (Actinidia chinensis) plants caused by P. savastanoi and, in particular, no in-depth studies of the complete genome sequence or pathogenic mechanism, long-lasting and environmentally friendly control measures against this pathogen in kiwifruit are lacking. This study therefore has both theoretical value and practical significance.

Results: We report the complete genome sequence of P. savastanoi strain MHT1, which was first reported as the pathogen causing bacterial canker in kiwifruit plants. The genome consists of a 6.00-Mb chromosome with 58.5\% GC content and 5008 predicted genes. Comparative genome analysis of four sequenced genomes of representative $P$. savastanoi strains revealed that 230 genes are unique to the MHT1 strain and that these genes are enriched in antibiotic metabolic processes and metabolic pathways, which may be associated with the drug resistance and host range observed in this strain. MHT1 showed high syntenic relationships with different $P$. savastanoi strains. Furthermore, MHT1 has eight conserved effectors that are highly homologous to effectors from P. syringae, Pseudomonas amygdali, and Ralstonia solanacearum strains. The MHT1 genome contains six genomic islands and two prophage sequences. In addition, 380 genes were annotated as antibiotic resistance genes and another 734 as encoding carbohydrate-active enzymes.
\end{abstract}

Conclusion: The whole-genome sequence of this kiwifruit bacterial canker pathogen extends our knowledge of the P. savastanoi genome, sets the stage for further studies of the interaction between kiwifruit and P. savastanoi, and provides an important theoretical foundation for the prevention and control of bacterial canker.

Keywords: Pseudomonas savastanoi, Kiwifruit bacterial canker, Comparative genomics, Effector, Genome

\section{Introduction}

Bacterial canker of kiwifruit (Actinidia spp.) is a serious threat to the kiwifruit industry that causes substantial crop losses worldwide [1]. After the first symptoms of canker disease (such as leaf spots) appear, the disease

*Correspondence: hqx99@163.com

${ }^{\dagger}$ Mingzhao Zhong and Yunhao Sun contributed equally to this work.

${ }^{6}$ Present Address: Guangzhou, People's Republic of China

Full list of author information is available at the end of the article spreads quickly to the rest of the plant, which can be destroyed within 1 year [2]. The main methods of disease control and mitigation include chemical treatment, orchard management, and breeding of resistant varietie, however, there are currently no effective methods to directly kill the causal pathogen [2]. Previous studies have reported that $P$. syringae pv. actinidiae is one of the main pathogenic agents of Actinidia spp. pathogenic bacterial canker disease [1-6]. 
P. syringae is an important phytopathogenic bacterium and one of the most relevant models for research on microbe-host interactions [7]. P. savastanoi is part of the $P$. syringae complex, which encompasses more than 60 pathovars, and P. savastanoi is recognized as a later synonyms of $P$. amygdali [8]. It has been reported that $P$. savastanoi could causes various diseases are on different host plants. P. savastanoi pv. savastanoi is usually reported as the causal agent of olive (Olea europaea) knot disease, which manifests during rainy months with moderate temperatures $\left(10-20^{\circ} \mathrm{C}\right)$ [9]; P. savastanoi pv. fraxini causes cankers accompanied by excrescences in European ash (Fraxinus excelsior) [10]; P. savastanoi pv. nerii induces knots in oleander (Nerium oleander), olive and ash [9]; P. savastanoi pv. retacarpa induces knots in broom (Retama sphaerocarpa) [9]; Pseudomonas savastanoi pv. mandevillae pv. nov., a clonal pathogen causing an emerging, devastating disease of the Ornamental plant Mandevilla spp. [11].

A growing number of $P$. savastanoi strains have been isolated and their genomes sequenced. Indeed, the National Center for Biotechnology Information (NCBI) database (https://www.ncbi.nlm.nih.gov/genome/) contains nearly 130 reports of genome assembly and annotation for $P$. savastanoi strains. This increasing amount of information highlights the biodiversity of $P$. savastanoi strains and has also helped facilitate a growing understanding of the pathogen's underlying pathogenic mechanisms, gene regulatory networks, and evolutionary processes $[9,12,13]$. However, no genome sequence of a $P$. savastanoi strain that causes bacterial canker in Actinidia spp. plants is currently available, underscoring the need for a more comprehensive and detailed comparative genomics analysis of $P$. savastanoi.

In this work, we sequenced the genome of $P$. savastanoi strain MHT1, which was isolated from an infected golden kiwifruit (Actinidia chinensis) plant. Further, by comparative evolutionary and genomics analyses with other sequenced $P$. savastanoi strains, we investigated host-specific candidate genes, conserved type III secretion system effector proteins, and their evolutionary relationships.

\section{Results}

\section{Isolation of pathogenic bacteria and pathogenicity tests}

Bacterial canker disease was observed in the kiwifruit cultivars 'HongYang' and 'WuZhi' at the Xiache kiwifruit plant base $\left(115^{\circ} 04^{\prime} \mathrm{N}, 24^{\circ} 64^{\prime} \mathrm{E}\right)$ in Heping County, Heyuan City, Guangdong Province, China. Symptoms such as cankers, cracks, and lesions with halos appeared on the leaves and trunks during early spring (Fig. 1A and B) under climatic conditions consisting of low temperatures, strong winds, and heavy rainfall. During year of
2019-2021, samples of disease leaves were collected the at three different time points. From the different samples, we isolated one same bacterium forming white colonies on plates (Fig. 1C), which was identified as pseudomonads by phylogenetic analysis by $16 \mathrm{~S}$ rDNA and $S y r B$ gene [14] (Figure S1). By The isolated strain reproduced typical symptoms on the leaves and tender buds of HongYang and WuZhi kiwifruit plants when infiltrated into the leaves (Fig. 1D and E) or spraying onto the abaxial sides of the leaves (Fig. 1F and G). We named this pathogenic strain MHT1, which is an acronym for the Chinese pronunciation of kiwifruit (Mi Hou Tao).

\section{Genome sequencing, assembly, and functional annotation}

To obtain more information about this pathogen and to explore the interaction mechanisms of the MHT1 strain with kiwifruit, we sequenced the MHT1 genome using the PacBio Sequel platform (Genedenovo Biotechnology Co., Ltd., Guangzhou, China). We obtained $1.12 \mathrm{~Gb}$ of reads representing an 187-fold coverage of the entire genome by SMRT sequencing (Genedenovo Biotechnology Co., Ltd., Guangzhou, China). After removing ambiguous and low-quality reads and adapters, we assembled the clean data into a single molecule of approximately $6.00 \mathrm{Mb}$ (Fig. 2A) with the program MECAT [15]. The circular chromosome harbored 5008 predicted genes and exhibited a GC content of $58.5 \%$ (Table 1). We used several complementary strategies to predict the number of different RNAs, clustered regularly interspaced short palindromic repeats (CRISPR), genomic islands (GIs), prophage sequences, interspersed repeats, tandem repeats, and transposons along the chromosome (Table 1 . The distribution statistics of the MHT1 gene complement against the Non-Redundant Protein Database (NR) (NCBI) showed that strain MHT1 shares 1949 and 1186 genes with P. syringae and P. savastanoi, respectively (Figure S2 and Table S1). According to the observed average nucleotide identity (ANI) (Figure S3), we refer to this strain as Pseudomonas savastanoi.

\section{Genetic relationship between MHT1 and other Pseudomonas savastanoi strains}

We performed a phylogenetic analysis between MHT1 and four representative sequenced strains of $P$. savastanoi that have not been reported to cause bacterial canker on kiwifruit (assemblies ASM1220v1, ASM16401v3, ASM1485547v1, and ASM1714087v1)(Table S2) based on their whole genomes using the REALPHY platform [16] (Fig. 2B). We also compared these five P. savastanoi strains and investigated their strain-specific and shared genes (Fig. 2C). We determined that 230 genes are unique to MHT1. We identified the extent of 


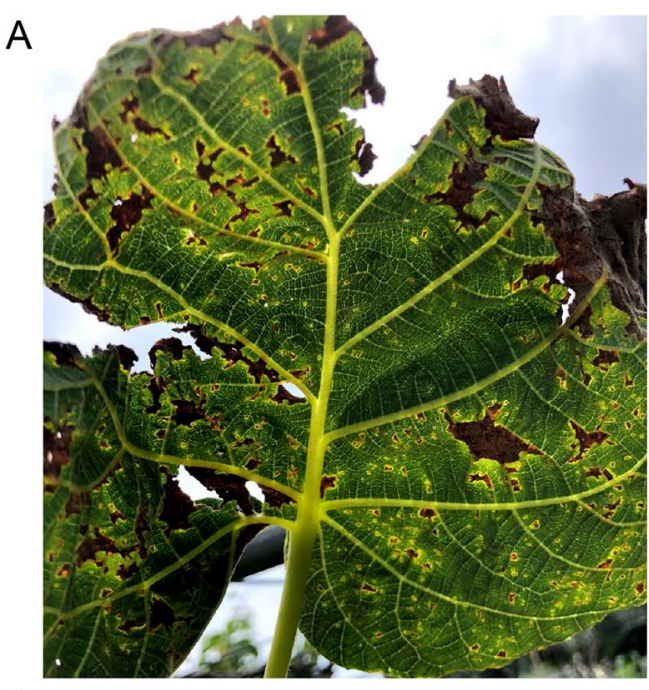

B T
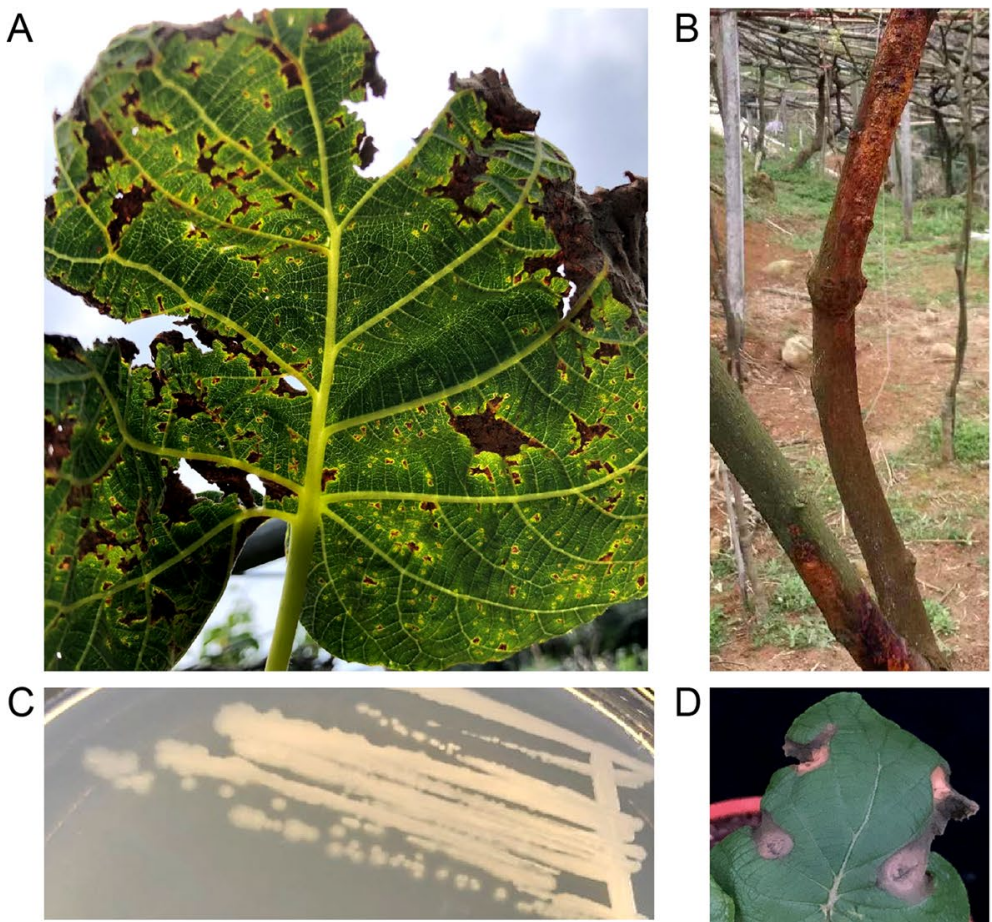

E
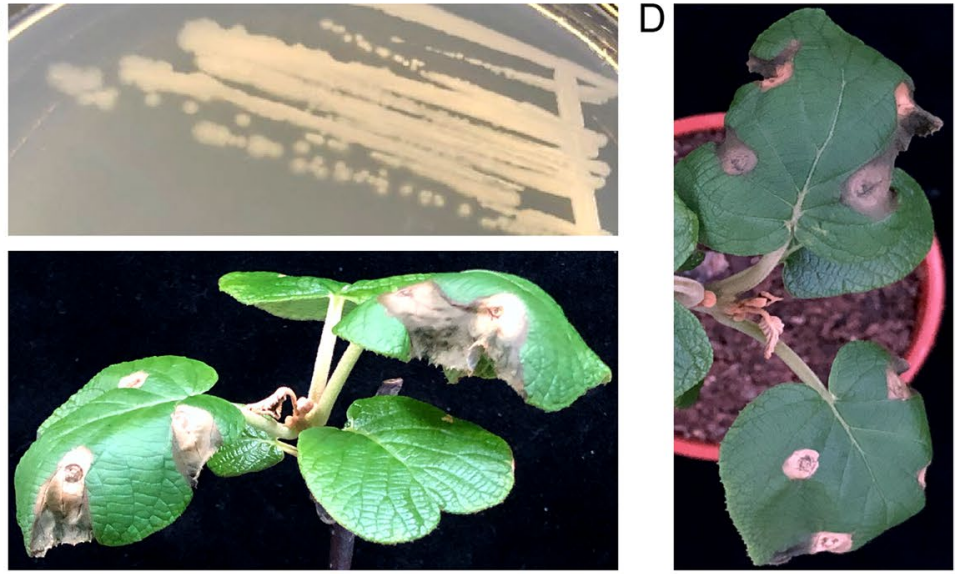

$\mathrm{F}$

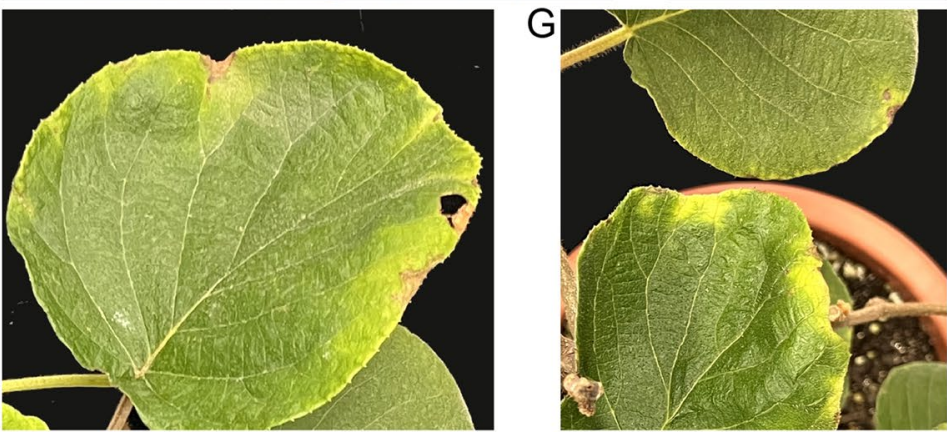

Fig. 1 Isolation and pathogenicity tests of Pseudomonas savastanoi strain MHT1. A and B. Representative leaf and stem of Actinidia chinensis plants with bacterial canker symptoms sampled from the Xiache kiwifruit plant base $\left(115^{\circ} 04^{\prime} \mathrm{N}, 24^{\circ} 64^{\prime} \mathrm{E}\right)$, Heping County, Heyuan City, Guangdong Province, China. Morphology of Pseudomonas savastanoi MHT1 colonies. A single colony of the MH1 strain was streaked and cultivated on LB medium at $28^{\circ} \mathrm{C}$ for $24 \mathrm{~h}$. D and E. Symptoms on the leaves and tender buds of A. chinensis plants (Hong Yang kiwifruit in C; WuZhi kiwifruit in $\mathbf{D}$ ) generated by P. savastanoi MHT1 strains 8 days post-inoculation. A. chinensis leaves were infiltrated with bacterial suspensions $\left(10^{8} \mathrm{CFU} / \mathrm{mL}\right.$ in $1 \mathrm{mM}$ $\mathrm{MgCl}_{2}$ ) and photographed 8 days later. CFU, colony-forming units. Negative control, leaves infiltrated with $10 \mathrm{mM} \mathrm{MgCl} 2 . \mathbf{F}$ and $\mathbf{G}$. Symptoms on the leaves of A. chinensis plants (HongYang kiwifruit) generated by P. savastanoi MHT1 strains 6 days post-infection. Abaxial side leaves of $A$. chinensis were sprayed with bacterial suspensions $\left(10^{8} \mathrm{CFU} / \mathrm{mL}\right.$ in $\left.1 \mathrm{mM} \mathrm{MgCl}\right)$ and photographed 6 days later

synteny between MHT1 and the four representative strains using the C-Sibelia program [17] (Fig. 3). Collinear blocks in the MHT1 genome accounted for $87.8 \%$ (versus ASM1485547v1), 87.2\% (versus ASM1220v1),
84.7\% (versus ASM1714087v1), and 82.8\% (versus ASM16401v3) of the total genome (Table 2).

We performed Gene Ontology (GO) [18] enrichment analysis to evaluate the possible function of these 


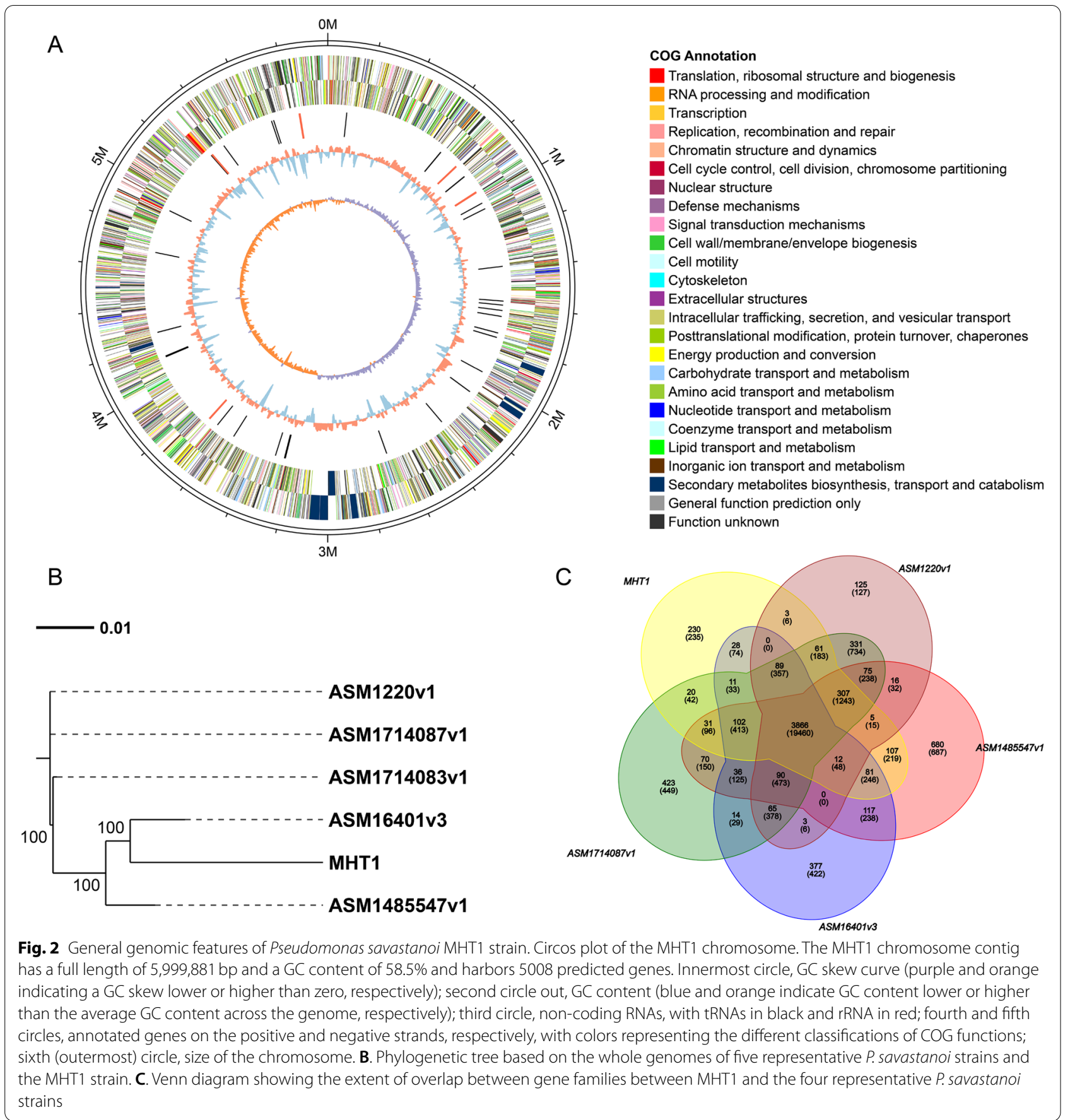

MHT1-specific genes: We observed enrichment for the GO terms 'antibiotic metabolic process' and 'drug metabolic process' (Fig. 4A). Subsequently, we performed a Kyoto Encyclopedia of Genes and Genomes (KEGG) [19] pathway analysis with the same MHT1-specific genes. The top enriched KEGG pathways were associated with 'pentose and glucuronate interconversions', 'metabolic pathways', 'amino sugar and nucleotide sugar metabolism, 'nonribosomal peptide structures', 'cysteine and methionine metabolism', and 'degradation of aromatic compounds' (Fig. 4B). These results showed that although MHT1 has high similarity with these four $P$. savastanoi strains, it still has many unique genes that are enriched in several physiological pathways. 
Table 1 General feature of the Pseudomonas savastanoi strain MHT1 genome

\begin{tabular}{ll}
\hline Features & Total \\
\hline Size (bp) & $5,999,881$ \\
G+C content (\%) & 58.47 \\
Coding genes & 5008 \\
tRNA & 63 \\
23S_rRNA & 5 \\
16S_rRNA & 5 \\
5S_rRNA & 6 \\
SRNA & 19 \\
CRISPR number & 6 \\
Genomic islands & 4 \\
Prophage & 2 \\
Interspersed repeats & 82 \\
Tandem repeats & 204 \\
Transposon & 2 \\
\hline
\end{tabular}

\section{Prediction and analysis of conserved type III effectors in Pseudomonas savastanoi strain MHT1}

Like many other gram-negative plant pathogenic bacteria, $P$. savastanoi may delivers many different type III effector proteins (T3Es) into host plants for its pathogenesis via a type III secretion system (T3SS) [13]. Given that different pathovars of $P$. savastanoi can infect several plant hosts, each strain may carry an array of conserved effector proteins that largely determines their host range [13]. To explore the characteristics of the MHT1 conserved effector protein arsenal, we searched for potential type III effectors encoded by the MHT1 genome using the T3E database and the program EffectiveT3 [20, 21]. We thus identified 26 proteins related to the T3SS and T3Es in the MHT1 genome (Table S3). We next looked for these T3Es in other $P$. syringae, $P$. amygdali, and Ralstonia solanacearum strains whose genomes have been sequenced and that can infect plant hosts [20]. We obtained eight conserved T3Es in MHT1 with strong similarity to the HopM1, AvrE1, and HopA1 effectors of P. syringae strains; the HopAH1and HopI1 effectors of $P$. amygdali strains; and the RipQ, RipW, and RipBG effectors of $R$. solanacearum strains (Table 3) [22-27]. We also predicted the subcellular localization of these potential effectors using Plant-mPLoc (http://www.csbio.sjtu. edu.cn/bioinf/plant-multi/) and Protein Homology/ analogY Recognition Engine V 2.0 (http://www.sbg. bio.ic.ac.uk/phyre2/html/page.cgi?id=index) (Table 3). These results suggest that the existence of several conserved effector proteins may be important to the pathogenicity of MHT1.

\section{Genomic islands and prophage elements}

Genomic islands (GIs) are fragments of DNA derived from horizontal gene transfer between different bacterial genomes [28]. We screened the genome of MHT1 for GIs using Island viewer software [29]. We identified six GIs in the genome (Fig. 5) with the IslandPath-DIMOB program [29]. Over the entire chromosome, the lengths of GIs ranged from 12,298 to $64,840 \mathrm{bp}$, with an average size of $26,497 \mathrm{bp}$ and covering a total length of $105,988 \mathrm{bp}$. These GIs contained 107 genes (Table S4), which mainly encoded FAD-binding oxidoreductases, short-chain dehydrogenase/reductase (SDR) family oxidoreductases, NAD(P)dependent alcohol dehydrogenases, ATP-dependent helicase $\mathrm{HrpB}$, glutathione $S$-transferases, transcriptional regulators, and ATP binding cassette $(\mathrm{ABC})$ transporter permeases.

Identification of prophages is important for the study of the genome of the MHT1 strain and its genetic potential [30]. In this study, we established that the MHT1 genome harbors two prophage regions (Table S5) with a total size of 55,399 bp using the program Phage_Finder [31]. The lengths of prophage regions 1 and 2 were 25,508 bp (from bp $5,334,617$ to $5,360,124$, with a GC content of $59.4 \%$ ) and 29,891 bp (from bp 5,702,675 to 5,732,565, with a GC content of 58.1\%), respectively. Sixty-five protein-coding genes were predicted in these two prophage sequences, encoding lysozymes, phage tail proteins, lipoproteins, glycoside hydrolases, and terminases (Table S6).

\section{Carbohydrate-active enzymes, antibiotic resistance, and substitution rate $\left(K_{\mathrm{a}} / K_{\mathrm{s}}\right)$ analysis}

Carbohydrate-active enzymes (CAZymes) are proteins that break down carbohydrates into smaller molecules by forming, hydrolyzing, and modifying glycosidic bonds [32]. In this study, we identified 734 genes annotated as CAZyme gene family members in the MHT1 genome (Figure S4). Among them, glycoside hydrolases and glycosyl transferases were the two most abundant protein families, followed by carbohydrate-binding modules, carbohydrate esterases, enzymes with auxiliary activities, and polysaccharide lyases.

We further analyzed antibiotic resistance genes (ARGs) in the MHT1 genome via the Comprehensive Antibiotic Resistance Database (CARD) [33]. We identified 380 ARGs (Table S7); these genes were potentially involved in resistance to fluoroquinolone, polyamine, macrolide, fosfomycin, aminoglycoside, pyrazinamide, cephalosporin, 


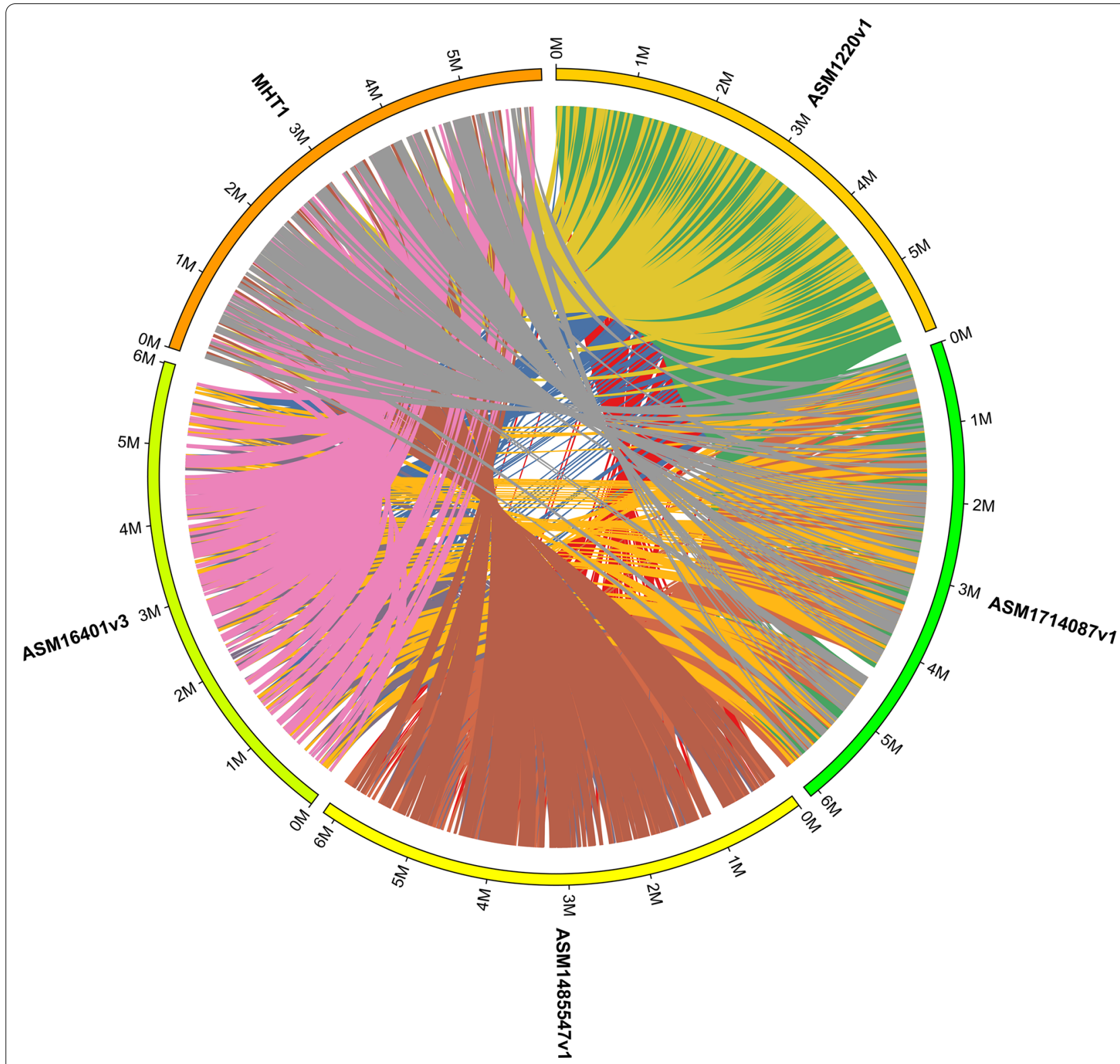

Fig. 3 Synteny map of the genomes of MHT1 and four representative P. savastanoi strains: ASM1220v1, ASM1714087v1, ASM1485547V1, and ASM16401v3

Table 2 Comparison of collinearity between MHT1 and other strains of Pseudomonas savastanoi

\begin{tabular}{ll}
\hline Strain & $\begin{array}{l}\text { Collinear with } \\
\text { SY1 (\%) }\end{array}$ \\
\hline ASM1485547v1 & 87.82 \\
ASM1220v1 & 87.24 \\
ASM1714087v1 & 84.70 \\
ASM16401v3 & 82.82 \\
\hline
\end{tabular}

isoniazid, isoniazid, elfamycin, ethionamide, lincosamide, glycopeptide, and rifamycin.

To estimate the evolutionary dynamics of $P$. savastanoi, we employed the MUMmer 3 package [34] to determine orthologous genes between the five strains and calculated the ratio between nonsynonymous mutation rate $\left(K_{\mathrm{a}}\right)$ and synonymous mutation rate $\left(K_{\mathrm{s}}\right)$ for each gene by the free ratio model [35]. Only four genes showed $K_{\mathrm{a}} / K_{\mathrm{s}}$ values of $>0.5$ (indicative of positive selection); these genes encoded a DUF2244 domain-containing protein, an ATPase, a 

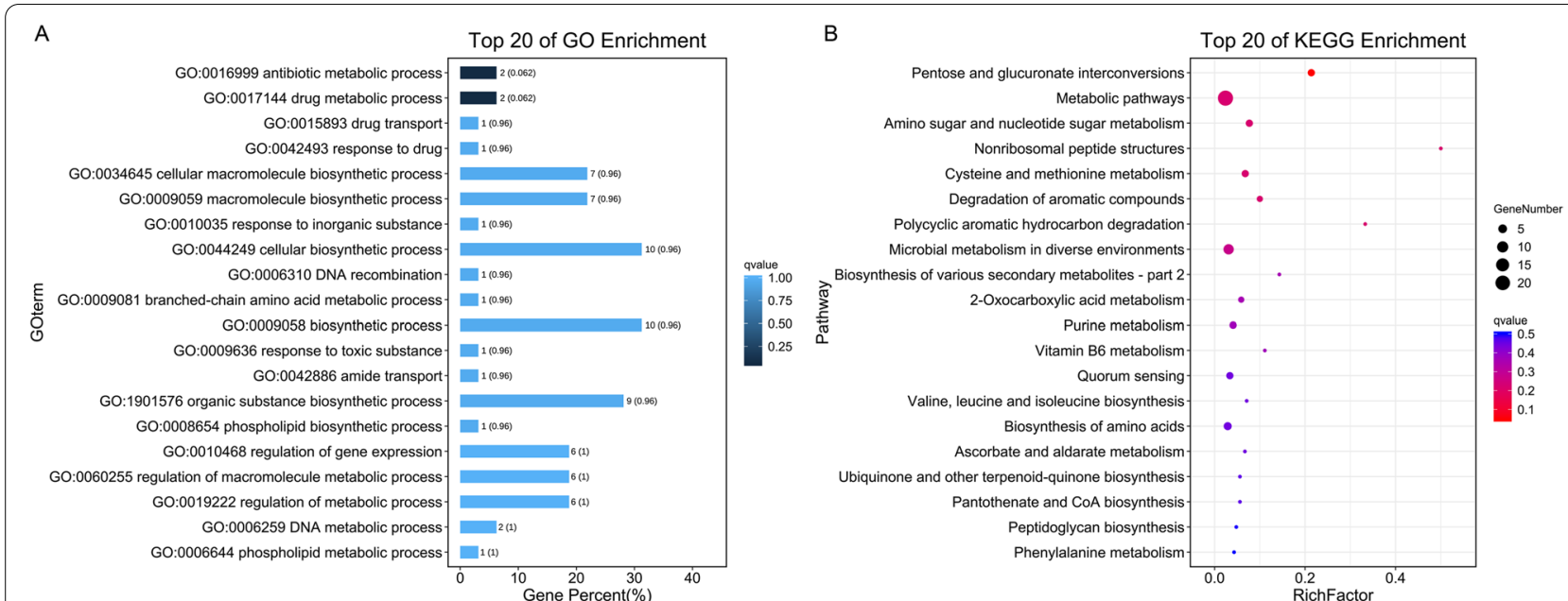

Fig. 4 Top 20 enriched GO and KEGG pathways for MHT1-specific genes compared to the ASM1220v1, ASM1714087V1, ASM1485547v1, and ASM16401v3 strains. A, Enriched GO categories. B, Enriched KEGG pathways

(2Fe-2S)-binding protein, and a BON (bacterial OsmY and nodulation) domain-containing protein (Table S8).

\section{Discussion}

Although at least 126 whole genomes of $P$. savastanoi strains have been sequenced, assembled, annotated, and uploaded to the NCBI database, none represent a strain that was isolated from an infected kiwifruit plant. $P$. savastanoi strains have been reported to mainly infect woody plants [9-13]. In this study, we demonstrated that kiwifruit, another woody plant, is also a natural host for this pathogen. We isolated P. savastanoi strain MHT1 from the leaves of kiwifruit plants that exhibited bacterial canker symptoms, from the Xiache kiwifruit plant base $\left(115^{\circ} 04^{\prime} \mathrm{N}, 24^{\circ} 64^{\prime} \mathrm{E}\right)$, Heping County, Heyuan City in Guangdong Province, China. This disease has recently emerged at this location, often showing outbreaks in the spring under rainy and low-temperature weather conditions. So far, from the aspect of disease epidemiology, we still don't know what is the pathogen transmission vector. However, given changes in local cultivation conditions due to climate change, we do not rule out the possibility that strain MHT1 may an opportunistic pathogenic pathogen of kiwifruit plant.

We sequenced and analyzed the genome of this new strain, providing an essential research basis for studying the characteristics of $P$. savastanoi strains in kiwifruit by allowing an examination of the molecular mechanisms behind the interaction between $P$. savastanoi strain and kiwifruit, with the goal to develop targeted biological control methods. We also selected four additional virulent $P$. savastanoi strains for study.
Phylogenetic analysis of MHT1 and these four strains indicated that MHT1 belongs to P. savastanoi and exhibits high homology with the genomes of the other four strains. However, the MHT1 strain also differed from the other representative strains by many genes. Notably, two GO terms, 'antibiotic metabolic process' and 'drug metabolic process', were enriched among genes unique to the MHT1 strain, indicating that it may have strong drug resistance potential. This observation suggests that screening specific bactericidals will be a prerequisite to the effective prevention and control of the diseases caused by MHT1 in production practice. In agreement with this, we identified up to 380 ARGs (Table S7) in the MHT1 genome, further supporting the speculation that the MHT1 strain may exhibit strong resistance to various antibiotics and other chemicals.

In addition, by KEGG pathway enrichment, we determined that the genes unique to the MHT1 strain are mainly involved in the pathways 'pentose and glucuronate interconversions,' 'metabolic pathways', 'amino sugar and nucleotide sugar metabolism', 'nonribosomal peptide structures', 'cysteine and methionine metabolism', and 'degradation of aromatic compounds', which may function during infections of kiwifruit and survival in certain environments, such as low temperature and high humidity. It remains to be determined whether the MHT1 strain, like other P. savastanoi strains, has a strong ability to infect other woody hosts or whether it can only infect plants in the kiwifruit production areas where it was initially isolated. We noticed the presence of 734 genes in the MHT1 genome that were annotated as CAZyme family genes (Figure S4), among which 


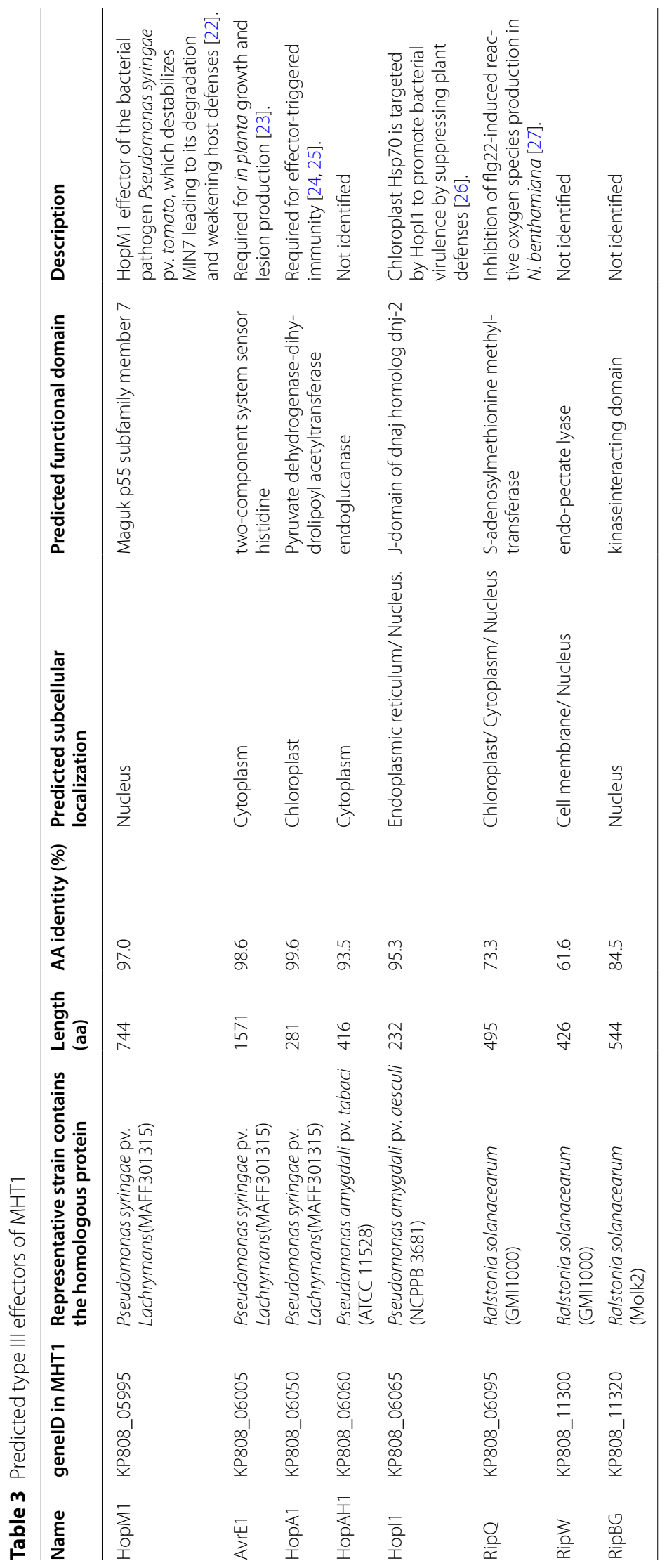




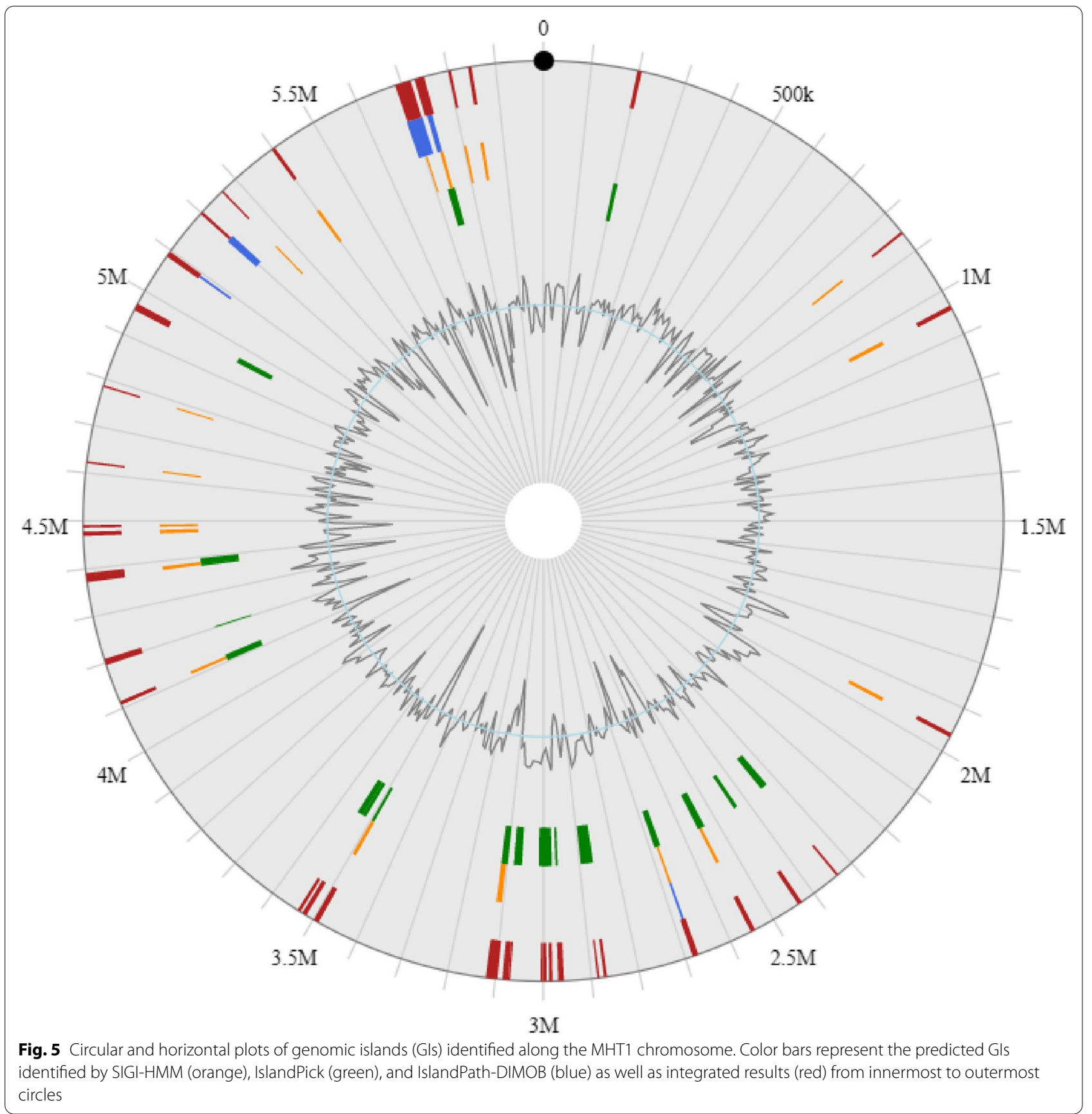

glycoside hydrolases and glycosyl transferases were the two most abundant families. Whether this feature helps MHT1 adapt to the host plant and environment will require further research to elucidate.

In this study, we investigated conserved type III effectors in P. savastanoi MHT1 strains and found that MHT1 presents three effectors that are highly homologous to the RipQ, RipW, and RipBG effectors of $R$. solanacearum strains. This implies that there may be effectors with similar functions in different gram-negative plant pathogenic bacteria and that these effectors may play important roles in the infection of the host, explaining their retention over the course of evolution. The effectors may also have been obtained by horizontal gene transfer, which could provide a molecular basis for MHT1 to acquire stronger pathogenicity or the ability infect more host plants. 


\section{Conclusions}

Here, we reported on the complete genome sequence of the $P$. savastanoi strain MHT1, isolated from infected kiwifruit plants showing bacterial canker symptoms. Comparative genomic analysis with $P$. savastanoi strains indicated that the MHT1 genome harbors 230 unique genes enriched in antibiotic metabolism pathways. In addition, 380 and 734 genes in the genome of the MHT1 strain were annotated as antibiotic resistance genes or as encoding carbohydrate-active enzymes, respectively. Furthermore, MHT1 has eight conserved effectors that are highly similar to other effectors from $P$. syringae, P. amygdali, and $R$. solanacearum strains. Collectively, these results provide a solid foundation for further studying the host specificity of MHT1 and for screening effective antibiotics for the prevention and control of bacterial canker caused by this pathogen.

\section{Materials and methods Preparation of strains}

Pseudomonas savastanoi strain MHT1 was isolated from the leaves of one Actinidia chinensis plant that exhibited bacterial canker symptoms, on the Xiache kiwifruit plant base $\left(115^{\circ} 04^{\prime} \mathrm{N}, 24^{\circ} 64^{\prime} \mathrm{E}\right)$, Heping County, Heyuan City, Guangdong Province of China. Species identification was performed by morphological and molecular analyses. Single colonies were selected after 1 day of culture in $\mathrm{LB}$ medium $(10 \mathrm{~g} \mathrm{NaCl}, 10 \mathrm{~g}$ peptone, $10 \mathrm{~g}$ agar, and $5 \mathrm{~g}$ yeast extract, dissolved in $1 \mathrm{~L}$ water, $\mathrm{pH} 7.0$ ) at $28^{\circ} \mathrm{C}$. The selected clones were grown in LB liquid medium for $18 \mathrm{~h}$ at $28^{\circ} \mathrm{C}$, followed by centrifugation at $4500 \mathrm{r} / \mathrm{min}$ for 5 min to collect cells. Phylogenetic trees were constructed by using software MEGA7.0, according to (NJ) neighbor joining method, the bootstrap replications are 1000.

\section{Pathogenicity tests}

MHT1 cells were cultured in LB medium at $28^{\circ} \mathrm{C}$ for $24 \mathrm{~h}$, harvested by centrifugation, and adjusted to $10^{8} \mathrm{CFU} /$ $\mathrm{mL}$ in $10 \mathrm{mM} \mathrm{MgCl}_{2}$. The suspension was infiltrated into the leaves of Actinidia chinensis plants (HongYang kiwifruit and WuZhi kiwifruit) with a needleless syringe. The negative control consisted of infiltration with $1 \mathrm{mM}$ $\mathrm{MgCl}_{2}$ without bacteria. For the leaf spraying infection test, the suspension was sprayed onto the abaxial sides of the leaves of Actinidia chinensis plants (HongYang kiwifruit). After infection, plants were grown under $100 \mu \mathrm{mol} \cdot \mathrm{m}^{-2} \cdot \mathrm{s}^{-1}$ light irradiance with a 12 -h-light/12h-dark $\left(20^{\circ} \mathrm{C} / 18^{\circ} \mathrm{C}\right)$ cycle in $95 \%$ relative humidity. Photographs were taken 8 days post-inoculation. The pathogenic bacteria were isolated and identified from the diseased tissues of HongYang kiwifruit and WuZhi kiwifruit.
DNA extraction, genome sequencing, and assembly Genomic DNA from P. savastanoi strain MHT1 was extracted with the TIANamp Bacteria DNA Kit (TIANGEN BIOTECH, Beijing, China) and quality-tested on a Qubit 2.0 Fluorometer (Life Technologies, Carlsbad, CA, USA). DNA concentration was measured on a Nanodrop (Thermo Fisher Scientific, Wilmington, USA).

Genome sequencing was performed on a PacBio longread sequencer (Pacific Biosciences, Menlo Park, CA, USA). SMRTbell libraries were obtained using g-TUBE (Covaris, Woburn, Massachusetts, USA) and end repair. According to the manufacturer's protocols of the Blue Pippin system (Pacific Biosciences), fragments with sizes larger than $10 \mathrm{~kb}$ were selected. The quality and average size of the fragments of the library were estimated using a Qubit 2.0 fluorometer (Life Technologies, Carlsbad, CA, USA) and Bioanalyzer 2100 (Agilent Technologies, Santa Clara, CA, USA). The PacBio Sequel system (Pacific Biosciences, Menlo Park, CA, USA) was used to perform SMRT sequencing. The resulting continuous long reads were used for de novo assembly with the Falcon program (version 0.3.0) [36].

\section{Functional genome annotation}

To predict the open reading frames (ORFs), the NCBI prokaryotic genome annotation pipeline and Prokka (version 1.11) were used [37, 38]. CRISPRfinder (version 4.2.17) was used to estimate CRISPR elements [39]. Ribosomal RNAs (rRNAs), small RNAs (sRNAs), and transfer RNAs (tRNAs) were predicted using the programs rRNAmmer (version 1.2), cmscan (version 1.1.2), and tRNAscan (version 1.3.1), respectively [40-42]. Tandem repeat elements were predicted with the program TRF (version 4.09) [43], interspersed repeat elements were predicted with RepeatMasker (version 4.0.5) [44], and transposons were predicted with TransposonPSI (version 1.0.0) (http://transposonpsi.sourceforge.net/) [45]. Based on sequence similarity, the predicted genes in strain MHT1 were annotated by BLASTN $\left(E\right.$-value $\left.<1 \mathrm{e}^{-5}\right)$, combined with analysis with Gene Ontology (GO), Cluster of Orthologous Groups of proteins (COG), Kyoto Encyclopedia of Genes and Genomes (KEGG), Swissport, and NCBI Non-Redundant Protein (NR) databases. Annotation of protein families was conducted based on Pfam_Scan (version 1.6) and the Pfam database (version 32.0) [46]. Prophages and GIs (gene islands) were predicted using the program Phage_Finder (version 2.0) [28] and Island Viewer (version 4.0) (http://www.pathogenom ics.sfu.ca/islandviewer/upload/) [31], respectively. Type III effectors in the MHT1 strain were predicted with the T3E database [20]. The phylogenetic tree was obtained by the REALPHY program (https://realphy.unibas.ch/realp 
hy/) using the whole genome of the MHT1 and other sequenced genomes of $P$. savastanoi strains as input. The Comprehensive Antibiotic Resistance Database (CARD) [33] and the Carbohydrate-Active enZYmes (CAZy) [47] database were used for advanced annotations. The default parameters were used in all analyses.

\section{Identification of orthologous genes}

The alignment of the MHT1 genome and four other representative $P$. savastanoi strains was performed in an all-against-all comparison with the MUMmer 3 package (version 3.3.3) (http://mummer.sourceforge.net/) with default parameters [48]. Orthologous gene clusters in the genomes were estimated with default parameters and with a combination of the programs OrthoMCL (version 2.0 ) and DIAMOND (parameters of $E$-value $<1 \mathrm{e}^{-5}$, query cover $>30 \%)[49,50]$. The core orthologs and putative proteins in MHT1 were aligned by BLASTP. The score of significant matched proteins of each pair was assigned using a $1 \times 10^{-7}$ cut-off value [51].

\section{Substitution rate estimation}

The KaKs_Calculator Toolbox software (using the free ratio model with default parameters) (version 2.0) [52] was used to calculate the nonsynonymous mutation rate $\left(K_{\mathrm{a}}\right)$ and synonymous mutation rate $\left(K_{\mathrm{s}}\right)$. The $K_{\mathrm{a}} / K_{\mathrm{s}}$ values that were higher than 0.5 [53] were considered genes under positive selection within the $P$. savastanoi strains.

\section{Abbreviations \\ T3SS: Type III secretion system; T3E: Type III effector protein; NR: Non-Redun- dant Protein database; GO: Gene Ontology; KEGG: Kyoto Encyclopedia of Genes and Genomes; GI: Genomics island; CAZyme: Carbohydrate-active enzyme; ARG: Antibiotic resistance gene; CARD: Comprehensive Antibiotic Resistance Database.}

\section{Supplementary Information}

The online version contains supplementary material available at https://doi. org/10.1186/s12866-022-02459-4.

\footnotetext{
Additional file 1: Table S1. List of blast with Non-Redundant Protein Database (NR) of strain MHT1. Table S2. List of four representative sequenced strains of $P$. savastanoi. Table S3. List of identified genes related to type III effectors and the type III secretion system in the MHT1 genome. Table S4. List of genes in genomic islands (Gls) of the MHT1 genome. Table S5. Identification of prophages in the MHT1 genome. Table S6. List of genomic islands (GIs) identified in the MHT1 genome. Table S7. Identification of antibiotic resistance genes (ARGs) in the MHT1 genome. Table S8. List of genes with a $K_{a} / K_{s}$ values greater than 0.5. Figure S1. The Molecular identification of strain MHT1. A and B, the PCR products of the 165 rDNA and SyrB gene. M: Marker DNA; 1-5, five duplicate samples. C and $D$, the PCR products in $A$ and $B$ were retrieved and sequenced. Phylogenetic trees were constructed by using software MEGA7.0, according to (NJ) neighbor joining method, the bootstrap replications are 1000. Figure S2. Summary of species distribution of homologs in the Non-Redundant Protein Database (NR). Distribution of different homologous genes, as
}

determined by the NR database. The number of genes involved in the corresponding families is given in parentheses after the species name. Figure S3. The hot-map of average nucleotide identity (ANI) between the strain MHT1 and other four strains. Based on the completion of collinearity alignment, pyani [10] was used to calculate the average nucleotide homology of two genome alignment regions between the target genome and the reference related genome. Figure S4. Classification of CAZymes by the Carbohydrate-Active enZYmes Database. The horizontal axis represents the function classification of enzymes, and the vertical axis represents the number of encoding genes included in the classification.

\section{Acknowledgments}

We thank the Fruit Research Institute and Kiwi modern agricultural industrial park of Heping for their support in assisting with data analysis. We appreciate Guangzhou Genedenovo Biotechnology Co., Ltd. for technical support.

\section{Authors' contributions}

Mingzhao Zhong and Yunhao Sun conceived and designed the study. Mingzhao Zhong, Yunhao Sun, and Lina Xiong performed the experiments. Yunhao Sun, Xianzhi Zhang, and Hong Liang wrote the paper. Qunxin Han reviewed and edited the manuscript. All authors read and approved the manuscript.

\section{Authors' information}

Not applicable.

\section{Funding}

This work was supported by Funds for the construction of agricultural pest ecological control team (grant no. KA21031H104 to Qunxin Han, for the analysis of the data and writing of the manuscript), the Forestry Science and Technology Innovation Project of Guangdong Province (grant no.

2021KJCX015 to Xianzhi Zhang, for data analysis and writing the manuscript) and the Key Scientific and Technological Innovation Projects of Guangdong Forestry Bureau (grant no. 2020KJCX009 to Yunhao Sun, for the collection of data), the National Natural Science Foundation of China (grant no. 32000086 to Yunhao Sun, for the analysis of the data and writing of the manuscript), the Applied Basic Research Programs of Science and Technology Commission Foundation of Guangdong Province (grant no. 2019A1515110593 to Yunhao Sun, for the analysis of the data and writing of the manuscript), the Research Project of Innovative Institute for Plant Health from Zhongkai University of Agriculture and Engineering (grant no. KA21031H103 to Yunhao Sun, for the collection of data), and the Starting Research Fund from Zhongkai University of Agriculture and Engineering (grant no. KA200540844 to Yunhao Sun, for the collection of data).

\section{Availability of data and materials}

All data generated and analyzed in this study are included in this published article and the supplementary materials. The raw genome sequencing data for P. savastanoi strain MHT1 were deposited at GenBank under the BioProject ID: PRJNA736824 (https://www.ncbi.nlm.nih.gov/bioproject/PRJNA736824) and BioSample: SAMN19665368 (https://www.ncbi.nlm.nih.gov/biosample/ SAMN19665368/).

\section{Declarations}

Ethics approval, accordance and consent to participate Not applicable.

\section{Consent for publication}

Not applicable.

\section{Competing interests}

The authors declare that they have no competing interests.

\section{Author details}

${ }^{1}$ Innovative Institute for Plant Health, Zhongkai University of Agriculture and Engineering, Guangzhou, China. ${ }^{2}$ College of Resources and Environment, Zhongkai University of Agriculture and Engineering, Guangzhou, China. ${ }^{3}$ College of Agriculture and Biology, Zhongkai University of Agriculture 
and Engineering, Guangzhou, China. ${ }^{4}$ Guangdong Zhongkai Technology Development Co., Ltd, Huizhou, China. ${ }^{5}$ School of Life Sciences, Sun Yat-sen University, Guangzhou, China. ${ }^{6}$ Present Address: Guangzhou, People's Republic of China.

Received: 25 October 2021 Accepted: 25 January 2022 Published online: 04 February 2022

\section{References}

1. Vanneste JL. The scientific, economic, and social impacts of the New Zealand outbreak of bacterial canker of kiwifruit (pseudomonas syringae pv. actinidiae). Annu Rev Phytopathol. 2017;55:377-99.

2. Mazzaglia A, Studholme DJ, Taratufolo MC, Cai R, Almeida NF, Goodman T, et al. Pseudomonas syringae pv. actinidiae (PSA) isolates from recent bacterial canker of kiwifruit outbreaks belong to the same genetic lineage. PLoS One. 2012:7(5):e36518.

3. Luti S, Campigli S, Ranaldi F, Paoli P, Pazzagli L, Marchi G. Lsc $\beta$ and Iscy, two novel levansucrases of pseudomonas syringae pv. actinidiae biovar 3, the causal agent of bacterial canker of kiwifruit, show different enzymatic properties. Int J Biol Macromol. 2021;179:279-91.

4. Vandelle E, Colombo T, Regaiolo A, Maurizio V, Libardi T, Puttilli MR, et al. Transcriptional profiling of three pseudomonas syringae pv. actinidiae biovars reveals different responses to apoplast-like conditions related to strain virulence on the host. Mol Plant Microbe Interact. 2021;34(4):376-96.

5. Chapman JR, Taylor RK, Weir BS, Romberg MK, Vanneste JL, Luck J, et al. Phylogenetic relationships among global populations of pseudomonas syringae pv. actinidiae. Phytopathology. 2012;102(11):1034-44.

6. Scortichini M, Marcelletti S, Ferrante P, Petriccione M, Firrao G. Pseudomonas syringae pv. actinidiae: a re-emerging, multi-faceted, pandemic pathogen. Mol Plant Pathol. 2012;13(7):631-40.

7. Xin XF, Kvitko B, He SY. Pseudomonas syringae: what it takes to be a pathogen. Nat Rev Microbiol. 2018;16(5):316-28.

8. Gomila M, Busquets A, Mulet M, García-Valdés E, Lalucat J. Clarification of taxonomic status within the pseudomonas syringae species group based on a phylogenomic analysis. Front Microbiol. 2017;8:2422.

9. Moreno-Pérez A, Pintado A, Murillo J, Caballo-Ponce E, Tegli S, Moretti C, et al. Host range determinants of pseudomonas savastanoi Pathovars of Woody hosts revealed by comparative genomics and cross-pathogenicity tests. Front Plant Sci. 2020;11:973.

10. Caballo-Ponce E, Murillo J, Martínez-Gil M, Moreno-Pérez A, Pintado A, Ramos C. Knots untie: molecular determinants involved in knot formation induced by pseudomonas savastanoi in Woody hosts. Front Plant Sci. 2017:8:1089.

11. Caballo-Ponce E, Pintado A, Moreno-Pérez A, Murillo J, Smalla K, Ramos C. Pseudomonas savastanoi pv. Mandevillae pv. nov., a clonal pathogen causing an emerging, devastating disease of the ornamental plant Mandevilla spp. Phytopathology. 2021;111(8):1277-88.

12. Ramos C, Matas IM, Bardaji L, Aragón IM, Murillo J. Pseudomonas savastanoi pv. savastanoi: some like it knot. Mol Plant Pathol. 2012;13(9):998-1009.

13. Moreno-Pérez A, Ramos C, Rodríguez-Moreno L. HrpL regulon of bacterial pathogen of Woody host pseudomonas savastanoi pv. savastanoi NCPPB 3335. Microorganisms. 2021;9(7):1447.

14. Xiao CL, Chen Y, Xie SQ, Chen KN, Wang Y, Han Y, et al. MECAT: fast mapping, error correction, and de novo assembly for single-molecule sequencing reads. Nat Methods. 2017;14(11):1072-4.

15. Oksel C, Avin FA, Mirik M, Baysal-Gurel F. Identification and genetic characterization of pseudomonas syringae pv. syringae from sweet cherry in Turkey. Plant Dis. 2021. https://doi.org/10.1094/PDIS-10-21-2241-RE Online ahead of print.

16. Bertels F, Silander OK, Pachkov M, Rainey PB, van Nimwegen E. Automated reconstruction of whole-genome phylogenies from shortsequence reads. Mol Biol Evol. 2014;31(5):1077-88.

17. Minkin I, Pham H, Starostina E, Vyahhi N, Pham S. C-Sibelia: an easy-to-use and highly accurate tool for bacterial genome comparison. F1000Research. 2013;2(258):1-6.

18. Pomaznoy M, Ha B, Peters B. GOnet: a tool for interactive gene ontology analysis. BMC Bioinformatics. 2018;19(1):470.
19. Ogata H, Goto S, Sato K, Fujibuchi W, Bono H, Kanehisa M. KEGG: Kyoto encyclopedia of genes and genomes. Nucleic Acids Res. 1999:27(1):29-34

20. Peeters N, Carrère S, Anisimova M, Plener L, Cazalé AC, Genin S. Repertoire, unified nomenclature and evolution of the type III effector gene set in the Ralstonia solanacearum species complex. BMC Genomics. 2013:14:859.

21. Jehl MA, Arnold R, Rattei T. Effective--a database of predicted secreted bacterial proteins. Nucleic Acids Res. 2011;39(Database issue):D591-5.

22. Nomura K, Debroy S, Lee YH, Pumplin N, Jones J, He SY. A bacterial virulence protein suppresses host innate immunity to cause plant disease. Science. 2006;313(5784):220-3.

23. Jayaraman J, Yoon M, Applegate ER, Stroud EA, Templeton MD. AvrE1 and HopR1 from pseudomonas syringae pv. actinidiae are additively required for full virulence on kiwifruit. Mol Plant Pathol. 2020;21(11):1467-80.

24. Dahale SK, Ghosh D, Ingole KD, Chugani A, Kim SH, Bhattacharjee S. HopA1 effector from pseudomonas syringae pv syringae strain 61 affects NMD processes and elicits effector-triggered immunity. Int J Mol Sci. 2021;22(14):7440.

25. Kang $H$, Nguyen QM, Iswanto ABB, Hong JC, Bhattacharjee S, Gassmann W, et al. Nuclear localization of HopA1 (Pss61) is required for effectortriggered immunity. Plants (Basel, Switzerland). 2021;10(5):888.

26. Jelenska J, Yao N, Vinatzer BA, Wright CM, Brodsky JL, Greenberg JT. A $J$ domain virulence effector of pseudomonas syringae remodels host chloroplasts and suppresses defenses. Curr Biol. 2007;17(6):499-508.

27. Nakano M, Mukaihara T. Comprehensive identification of PTI suppressors in type III effector repertoire reveals that Ralstonia solanacearum activates Jasmonate signaling at two different steps. Int J Mol Sci. 2019;20(23):5992.

28. Rodriguez-Valera F, Martin-Cuadrado AB, López-Pérez M. Flexible genomic islands as drivers of genome evolution. Curr Opin Microbiol. 2016:31:154-60.

29. Bertelli C, Laird MR, Williams KP, Lau BY, Hoad G, Winsor GL, et al. IslandViewer 4: expanded prediction of genomic islands for larger-scale datasets. Nucleic Acids Res. 2017;45(W1):W30-w35.

30. Bondy-Denomy J, Qian J, Westra ER, Buckling A, Guttman DS, Davidson $A R$, et al. Prophages mediate defense against phage infection through diverse mechanisms. ISME J. 2016;10(12):2854-66.

31. Fouts DE. Phage_Finder: automated identification and classification of prophage regions in complete bacterial genome sequences. Nucleic Acids Res. 2006;34(20):5839-51.

32. Montgomery AP, Xiao K, Wang X, Skropeta D, Yu H. Computational Glycobiology: mechanistic studies of carbohydrate-active enzymes and implication for inhibitor design. Adv Protein Chem Struct Biol. 2017;109:25-76.

33. Alcock BP, Raphenya AR, Lau TTY, Tsang KK, Bouchard M, Edalatmand A, et al. CARD 2020: antibiotic resistome surveillance with the comprehensive antibiotic resistance database. Nucleic Acids Res. 2020:48(D1):D517-d525.

34. Hurst LD. The Ka/Ks ratio: diagnosing the form of sequence evolution. Trends Genet. 2002;18(9):486

35. Li J, Zhang Z, Vang S, Yu J, Wong GK, Wang J. Correlation between Ka/Ks and $\mathrm{Ks}$ is related to substitution model and evolutionary lineage. J Mol Evol. 2009;68(4):414-23.

36. Chin CS, Peluso P, Sedlazeck FJ, Nattestad M, Concepcion GT, Clum A, et al. Phased diploid genome assembly with single-molecule real-time sequencing. Nat Methods. 2016;13(12):1050-4.

37. Seemann T. Prokka: rapid prokaryotic genome annotation. Bioinformatics (Oxford, England). 2014;30(14):2068-9.

38. Tatusova T, DiCuccio M, Badretdin A, Chetvernin V, Nawrocki EP, Zaslavsky $L$, et al. NCBI prokaryotic genome annotation pipeline. Nucleic Acids Res. 2016:44(14):6614-24.

39. Grissa I, Vergnaud G, Pourcel C. CRISPRFinder: a web tool to identify clustered regularly interspaced short palindromic repeats. Nucleic Acids Res. 2007;35(Web Server issue):W52-7.

40. Lowe TM, Chan PP. tRNAscan-SE on-line: integrating search and context for analysis of transfer RNA genes. Nucleic Acids Res. 2016;44(W1):W54-7.

41. Lagesen $K$, Hallin $P$, Rødland EA, Staerfeldt HH, Rognes $T$, Ussery DW. RNAmmer: consistent and rapid annotation of ribosomal RNA genes. Nucleic Acids Res. 2007;35(9):3100-8.

42. Nawrocki EP, Eddy SR. Infernal 1.1: 100-fold faster RNA homology searches. Bioinformatics (Oxford, England). 2013;29(22):2933-5. 
43. Benson G. Tandem repeats finder: a program to analyze DNA sequences. Nucleic Acids Res. 1999;27(2):573-80.

44. Chen N. Using RepeatMasker to identify repetitive elements in genomic sequences. Curr Protoc Bioinformatics. 2004;25(4):1-14.

45. Vij S, Kuhl H, Kuznetsova IS, Komissarov A, Yurchenko AA, Van Heusden $\mathrm{P}$, et al. Chromosomal-level assembly of the Asian seabass genome using long sequence reads and multi-layered scaffolding. PLoS Genet. 2016;12(4):e1005954.

46. El-Gebali S, Mistry J, Bateman A, Eddy SR, Luciani A, Potter SC, et al. The Pfam protein families database in 2019. Nucleic Acids Res. 2019;47(D1):D427-d432.

47. Lombard V, Golaconda Ramulu H, Drula E, Coutinho PM, Henrissat B. The carbohydrate-active enzymes database (CAZy) in 2013. Nucleic Acids Res. 2014;42(Database issue):D490-5.

48. Kurtz S, Phillippy A, Delcher AL, Smoot M, Shumway M, Antonescu C, et al. Versatile and open software for comparing large genomes. Genome Biol. 2004;5(2):R12.

49. Buchfink B, Xie C, Huson DH. Fast and sensitive protein alignment using DIAMOND. Nat Methods. 2015;12(1):59-60.

50. Silva-Pereira TT, Ikuta CY, Zimpel CK, Camargo NCS, de Souza Filho AF, Ferreira Neto JS, et al. Genome sequencing of mycobacterium pinnipedii strains: genetic characterization and evidence of superinfection in a south American sea lion (Otaria flavescens). BMC Genomics. 2019:20(1):1030.

51. Hirsh $A E$, Fraser HB. Protein dispensability and rate of evolution. Nature. 2001;411(6841):1046-9.

52. Wang D, Zhang Y, Zhang Z, Zhu J, Yu J. KaKs_Calculator 2.0: a toolkit incorporating gamma-series methods and sliding window strategies. Genomics Proteomics Bioinformatics. 2010;8(1):77-80.

53. Wang Y, Yang L, Zhou K, Zhang Y, Song Z, He S. Evidence for adaptation to the Tibetan plateau inferred from Tibetan loach transcriptomes. Genome Biol Evol. 2015;7(11):2970-82.

\section{Publisher's Note}

Springer Nature remains neutral with regard to jurisdictional claims in published maps and institutional affiliations.

Ready to submit your research? Choose BMC and benefit from:

- fast, convenient online submission

- thorough peer review by experienced researchers in your field

- rapid publication on acceptance

- support for research data, including large and complex data types

- gold Open Access which fosters wider collaboration and increased citations

- maximum visibility for your research: over $100 \mathrm{M}$ website views per year

At $\mathrm{BMC}$, research is always in progress.

Learn more biomedcentral.com/submissions 